\title{
The Solution to Wheeler-DeWitt is Eight
}

\author{
E. Adi $^{1}$ and S. Solomon ${ }^{2,1}$ \\ 1 Permanent address Racah Institute of Physics \\ Hebrew University, 91904 Jerusalem, ISRAEL \\ eti@vms.huji.ac.il \\ sorin@vms.huji.ac.il \\ 2 Scuola Internazionale Superiore di Studi Avanzati \\ SISSA, Via Beirut n. 2-4, 34013 Trieste ITALY \\ sorin@tsmi19.sissa.it \\ Submitted to Phys. Lett. B.
}

\begin{abstract}
We describe a new geometrical solution of the Wheeler- DeWitt equation in two dimensional quantum gravity. The solution is the amplitude of a surface whose boundary consists of two tangent loops.

We further discuss a new method for estimating singular geometries amplitudes, which uses explicit recursive counting of discrete surfaces.
\end{abstract}




\section{Introduction}

In the quantization of gravity, wavefunctions are functions of the spatial metric and the other fields in the theory. The requirement of gauge invariance states that wavefunctions are required to obey the operator version of the space and time diffeomorphism constraints. The Weeler-DeWitt (WDW) equation expresses the invariance under the generator of time-diffeomorphisms [1] and plays a fundamental role in the theory [2].

For this reason it is very important to check for any candidate of quantum gravity, whether it engenders the WDW equation.

In two dimensions (2D) gravity the spatial geometry is completely specified by the length of the 1-D boundary, up to spatial diffeomorphisms. Therefore the minisuperspace approximation (considering spatial geometries that are specified by a single scalar factor) is exact.

In the absence of matter fields, the WDW equation in 2D becomes [3]:

$$
\left(-\left(l \frac{\partial}{\partial l}\right)^{2}+4 \mu l^{2}+\nu^{2}\right) \Psi(l)=0
$$

Where $\Psi$ are wavefunctions describing the the geometry of space-time. [BThe term $\nu^{2}$ is obtained by assuming that the states are associated to local operators in KPZ conformal theory [ [I] and assumes positive half-integer values..

$l$ is the length of the 1-D boundary , $\mu$ is the cosmological constant (which in the minisuperspace model is restricted be positive) and $\nu$ is a constant that can have positive half integer values in the case of pure gravity.

The solutions of this equation decaying at large lengths are the modified Bessel functions:

$$
\Psi_{O}(l) \sim K_{\nu}(2 \sqrt{\mu} l)
$$

It is quite nontrivial, but true that the same result is obtained by estimating with matrix model techniques appropriate path integral expressions.

In particular, the path integral over all the surfaces with one puncture, one marked point on the boundary, and disk topology (fig.1) was shown in [3] to correspond to the solution with $\nu=1 / 2$ of (11).

The physical interpretation of this integral is the amplitude of an universe of length $l$ propagated out of a cosmological constant insertion (geometrically represented as a puncture).

A second solution, corresponding to $\nu=\frac{3}{2}$ was shown to be obtained by applying the boundary operator on surfaces with disk topology [3]. The 
boundary operator measures the total length of the boundaries. When applied to a disk, it assumes the expectation value $l w(l)$ where $w(l)$ is the amplitude for the disk.

The path integral expressions solving the equation (西) for higher $\nu$ values do not have such a clear interpretation.

None of the two solutions mentioned above has the original HawkingHartle interpretation of being the amplitude of creating the universe from nothing [5]: the first one starts with an insertion, and the second one involves the measurement of a particular quantity (the boundary length).

The further search for purely geometric solutions to the WDW equation starting from "nothing" was impaired by the difficulties to estimate singular geometries amplitudes by KPZ or matrix model techniques.

Using a new discrete counting technique, we compute for the first time, the amplitude to propagate out of "nothing" an universe with the topology of two tangent circles, named in [6] "figure eight diagram".

It turns out that this amplitude fulfills the WDW equation (1) corresponding to $\nu=\frac{3}{2}$. We further discuss the geometrical interpretation of this solution.

We demonstrate the generality of our new combinatoric method by calculating in the last part of the letter another singular geometry example [7] unavailable by previous techniques.

\section{Disk amplitudes and their relation to WDW solutions}

The singular amplitudes computed in this paper are all related to the regular disk amplitude. Therefore, in order to describe their properties, we first recall

some known facts on the diagrams with disk topology in the matrix model framework.

Recall that the partition function of a disk with fixed area $A$ and perimeter $l$ has the form [3]:

$$
Z(A, l)=l^{\frac{1}{2}} A^{-\frac{5}{2}} e^{-\frac{l^{2}}{A}}
$$

The loop amplitude is defined to be:

$$
w(l)=\int Z(A, l) e^{-\mu A}
$$


and its value is found in [3] to be:

$$
w(l)=l^{-\frac{5}{2}} e^{-2 \sqrt{\mu} l}(1+2 \sqrt{\mu} l)
$$

The modified Bessel function $K_{\nu}$ admits an integral representation with an integrand similar in form to (3):

$$
K_{\nu}(z)=\frac{1}{2}\left(\frac{z}{2}\right)^{\nu} \int_{0}^{\infty} d t t^{-\nu-1} e^{-t-\frac{z^{2}}{4 t}} \quad, \quad|\arg z|<\frac{\pi}{2} \quad, \quad \operatorname{Re} z^{2}>0 .
$$

where $z=2 \sqrt{\mu} l$ and $\nu=\mu A$.

We thus learn that (4), (5) can be written in terms of the modified Bessel functions as:

$$
w(l)=l^{-1}(\sqrt{\mu})^{\frac{3}{2}} K_{\frac{3}{2}}(2 \sqrt{\mu} l)
$$

This result for the regular disk amplitude was found in [3] by means of semiclassical approximation combined with scaling arguments, as well as by matrix models results. However, it can also be obtained through direct counting of triangulate surfaces and by taking then the continuum limit [7].

In [3] it was argued that by construction, solutions of (1) obtained by conformal field theory KPZ techniques, are wavefunctions obtained by the insertion of local states operators. Solutions were sought therefore, by looking for wavefunctions of insertions of conformal operators on a disk. In pure gravity, the insertion of the cosmological constant is equivalent to marking a point on the disk. The explicit formula for the partition function of the marked (punctured) disk is: $A Z(A, l)$ where $Z(A, l)$ is given by (3)). After applying (雨) this gives for the marked disk wave function

$$
\Psi_{\text {punct. disk }}=(\sqrt{\mu})^{\frac{1}{2}} K_{\frac{1}{2}}(2 \sqrt{\mu} l)
$$

As observed in [3] this is according to (2) a solution of the 2D WDW equation (1). From the solutions achieved by inserting local conformal operators, this is in fact the only one which admits a simple geometrical picture.

By inserting the boundary operator, in the partition function, one obtains, as in [3] $l Z(A, l)$. By applying the transform in (4) this leads to the wave function :

$$
\Psi_{\text {boundary }}=(\sqrt{\mu})^{\frac{3}{2}} K_{\frac{3}{2}}(2 \sqrt{\mu} l)
$$


which according to (2) is also a solution of of the 2D WDW equation (11) with $\nu=3 / 2$.

The solution we present in the next section has the same functional form like (8) but it is obtained by considering the amplitude for surfaces whose boundary consists of two loops that coincide in one point.

This geometrical interpretation of the universe wave function as the amplitude for a universe originating "out of nothing" to split in tangent "circles" is a germane to the "foam" idea of Hawking and Hartle for the texture of the universe.

\section{Figure-eight}

Our technique to calculate the "figure-eight" and other singular geometry amplitudes is based upon exact counting of random triangulate surfaces [8]. We use a recursive equation which is obtained by expressing the change in the partition function of the discrete surface caused by removing one boundary edge.

While the use of the recursion equation is quite complicated in the nonsingular diagram case, in the singular diagram case, it consists of only one term which is usually related to an already known regular partition function.

It is lucky that the present method is complementary to the double limit matrix model in the sense that it needs the results of the matrix model for certain regular surfaces but it provides results for the singular geometries which are unavailable in the matrix model framework.

Here we present the continuous version of the argument chain and refere to [7, 9] for the detailed mathematical formulae which represent these manipulations in a rigorous quantitative way. The additional rigor [7, 9] is related to the fact that while in the present exposition we operate on unregularized (continuum) surfaces, in [7, 9], the lattice provides a well defined basis for counting possibilities.

As an example of the additional rigor provided by the discrete treatment, consider the following example. In the paragraphs below we use the fact that the number of ways in which one can split a continuum loop of length $l$ into two segments is proportional to $l$. In the lattice calculation this number is explicitly found to be $L-L_{o}+1$ where $L$ is the loop length, in lattice spacing units $a, L_{o}$ is the minimal length that two loops can have in lattice 
spacing units (i.e 6 in the case of triangulate lattice, 8 for a quartic lattice etc ...) and $L \geq L_{o}$. In addition to the technical advantages, the discrete triangulation formulation has methodological advantages. In fact much of the mathematical formulae expressing the amplitudes properties reported here and in [7, 9] were suggested to us by the direct simulation and visualization of RTS as reported in [10].

Let us now return to the estimation of the "figure-eight" amplitude. This is obtained by causing the disk boundary to assume the singular geometry shown in (fig.2). What is the partition function of such a geometry?

We first remind the reader that the loop amplitude $w(l)$ corresponds in the matrix model to the measurable $\operatorname{tr} \phi^{l / a}$ which creates a hole of length $l$ in a discrete surface with lattice spacing $a$. Its computation is made in the matrix model with the convention that one point of the loop is actually marked.

The unmarked amplitude $w^{m m}(l)$ [3] is related to the marked loop amplitude $w(l)$ by the relation: $w^{m m}(l)=\frac{w(l)}{l}$ which takes symmetry into consideration.

In order to deduce from a marked point loop amplitude the "figure-eight" amplitude, one marks another point on the loop and identify these two points. However, if the length of the loops is known (i.e $l_{1}, l_{2}$ ) then we need to mark only one point because the position of the second one is determined by $l_{1}$ and $l_{2}$. We conclude that:

$$
Z_{\text {fig. } 8}\left(A, l_{1}, l_{2}\right)=Z\left(A, l_{1}+l_{2}\right)
$$

and does not depend on either $l_{1}$ or $l_{2}$.

This result is more transparent in the context of triangulate surfaces (where in fact we found it initially). There, we have expressed by a recursive equation the partition function of a surface with two boundaries. The recursive formula sums all possibilities of removing one boundary edge. One of the terms we count is this "figure-eight" which is a two-loop surface but has the weight of the $L_{1}+L_{2}+1$ disk (fig.3). Thus (8) is written in triangulate lattice terms as:

$$
Z_{\text {fig.8 }}\left(N, L_{1}+L_{2}\right)=Z\left(N-1, L_{1}+L_{2}+1\right)
$$

where $N$ is the number of triangles (fig. 3). 
By taking into account all $L-5$ possibilities of dividing the $L(L \geq 6)$ loop into two (as explained above) we find that

$$
Z_{f i g .8}\left(N, L_{1}+L_{2}=L\right)=(L-5) * Z\left(N-1, L_{1}+L_{2}+1\right)
$$

which becomes in the continuum after applying (44) (using $a * L \rightarrow l, a^{2} *$ $N \rightarrow A \llbracket 11)$

$$
w_{\text {fig. } 8}(l)=(\sqrt{\mu})^{\frac{3}{2}} K_{\frac{3}{2}}(2 \sqrt{\mu} l)
$$

and is a solution of the WDW equation.

The equivalence between a disk with two marked boundary points and a "figure-eight", has a further geometrical meaning which was clarified to us by E. Witten. In order to discribe it, one can use fig. 4 . Consider a cut through the equator of a nodded torus. What we find is a continuum version of the triangulate diagram which appears in fig 3 . without specifying the values of the two loop lengths $l_{1}$ and $l_{2}$. Fig. 4 is equivalent, after removing the singularity, to a disk with two marked boundary points, just as the nodded torus is equivalent, after removing the singularity, to a sphere with two punctures.

\section{Singular geometries}

We can further use our knowledge to evaluate other singular geometries. These are obtained by passing from one topology to another. A "figure-eight" (fig. 3 ) is the evaluation of a two loop surface out of a disk. The "one handle figure-eight" (fig. 5) represents the evaluation of a one boundary torus out of a two loop surface. Its amplitude is the amplitude of the $l$ possible two loop surfaces of which it is decomposed. Using the result of [3] we have

$$
w\left(l_{1}, l_{2}\right)=\sqrt{l_{1}} \sqrt{l_{2}} \frac{e^{-2 \sqrt{\mu}\left(l_{1}+l_{2}\right)}}{l_{1}+l_{2}}
$$

we thus find for the "one handle figure-eight" (fig. 5):

$$
\bar{w}(l)=l e^{-2 \sqrt{\mu} l}
$$

The importance of singular geometries, as found in [7], is expressed by the role they play in the recursive relation description of partition functions of surfaces with more then one handle or boundary. 
Indeed, once the singular geometries are under control, one can contionously relate surfaces with different topologies by "smoothly" deforming them into one onother while passing through the appropriate singular topologies.

For instance the $k$ boundaries partition function is expressed by $k$ disklike terms corresponding to the $k$ loops, with additional "figure-eight"-like terms related to a $k-1$ loop surface. The $h$ handle and $k$ boundaries partition function is expressed as the $k$ boundaries partition function, with additional "one handle figure-eight"-like terms related to a surface with $h-1$ handles and $k+2$ loops.

This leads to a general strategy for the evaluation of surfaces: once a disk is formed it can dynamically change into other topology with one boundary or more. The change is done via singular geometries. It was drawn to our attention by N. Seiberg that using this picture one can expect to relate amplitudes of surfaces with various boundary topologies to the disk amplitude. The relation between the multi-loop and disk amplitudes was found by different techniques in [3].

We thank S. Elitzur, A. Goldberger, E. Rabinovici, N. Seiberg and E. Witten for discussions.

This work was supported in part by GIF (Germany-Israel Foundation) and by the Israeli Academy Foundation for Fundamental Research. 


\section{References}

[1] B,S. DeWitt, Phys. Rev. 160 (1967) 1113.

[2] S. Elitzur, A. Forge and E. Rabinovici, Phys. Lett. B289 (1992) 45.

[3] G. Moore, N. Seiberg, and M. Staudacher, Nucl. Phys. B362 (1991) 665.

[4] F. David, Mod. Phys. Lett.A3 (1988) 1651. J. Distler and H. Kawai, Nucl. Phys. B321 (1989) 509.

[5] J,B. Hartle and S,W. Hawking, Phys. RevD 28 (1983) 2960.

[6] P. Ginsparg and G. Moore, YCTP-p23-92, hep-th/9304011.

[7] E. Adi and S. Solomon, in preparation.

[8] W,T. Tutte, Can. J. Math 14 (1962) 21.

[9] E. Adi, M.Sc thesis (1993).

[10] E. Adi et. al., Phys. Lett. B320 (1994) 227.

[11] F. David, hep-th/9303127. 


\section{FIGURE CAPTIONS}

- Fig.1 A punctured disk.

- Fig.2 "Figure-eight".

- Fig.3 A triangulate "figure-eight" as a part of combinatorial counting.In the figure, $A_{N L_{1} L_{2}}$ is the number of possible two boundary triangulate surfaces with $N=10 \quad L_{1}=6 \quad L_{2}=4 . a_{N-1 L_{1}+L_{2}+1}$ is the number of disk topology triangulate surfaces composed of $N-1=9$ triangles and boundary length of $L_{1}+L_{2}+1=11$. The disk is the result of the removal of one boundary edge. (The one at the top of the diagram.)

- Fig.4 A cut through the equator of the nodded torus and the figureeight result. After the singularity is removed we find a disk with two marked boundary points.

- Fig.5 One-handle figure-eight. 
This figure "fig1-1.png" is available in "png" format from: http://arxiv.org/ps/hep-th/9404079v2 
This figure "fig1-2.png" is available in "png" format from: http://arxiv.org/ps/hep-th/9404079v2 
This figure "fig1-3.png" is available in "png" format from: http://arxiv.org/ps/hep-th/9404079v2 
This figure "fig1-4.png" is available in "png" format from: http://arxiv.org/ps/hep-th/9404079v2 
This figure "fig1-5.png" is available in "png" format from: http://arxiv.org/ps/hep-th/9404079v2 